Gynäkol Geburtsh Rundsch 1992;32(Suppl 1):66-67

\title{
Sektioindikation im Wandel
}

\begin{tabular}{|l|l|}
\hline W. & Gruber \\
\hline A. & Berger \\
\hline
\end{tabular}

II. Univers.-Frauenklinik Wien (Vorst.: Prof. Dr. H. Janisch)

Prof. Dr. W. Gruber, II. Univers.-Frauenklinik, Spitalgasse 23, A-1090 Wien

Weltweit beobachten Geburtshelfer ein Ansteigen der Kaiserschnittraten und befürchten, daß dieser Anstieg über ein sinnvolles Maß hinausgehen könnte.

Die Entscheidung zum Kaiserschnitt wird nicht nur von medizinischen Erwägungen des

Geburtshelfers getragen, sondern unterliegt auch vielfältigen Einflüssen von außer-halb der Geburtshilfe (Tab. 1).

Der Anstieg der Sektiofrequenz erfolgte etwa gleich-zeitig und gegenläufig mit dem erfreulichen Absinken der perinatalen Mortalität, die 1991 in Österreich 6,4\%0 betrug.

Wie O’Driscoll [1] bereits 1983 in seiner bemerkens-werten Studie zeigte, besteht jedoch keine direkte kausale Korrelation zwischen diesen beiden Entwicklungen.

Aus Gründen der mütterlichen Sicherheit versuchen Geburtshelfer, die Zahl der Kaiserschnitte so niedrig wie vertretbar zu halten und sind dabei unterschiedlich erfolg-reich.

Sektioraten in Wien 1991

Die Sektioraten der Wiener Entbindungsanstalten ran-gierten 1991 zwischen 4,7 und 20 \%, die Gesamtrate betrug 9,8 \%. Die Mehrzahl der Abteilungen führt etwa 10 \% Sec-tiones durch, die Universitätskliniken liegen mit je 12,5 \% verständlicherweise geringfügig höher.

2 Abteilungen, eine große öffentliche und eine mittel-große konfessionelle Abteilung liegen mit etwa $5 \%$ deut-

Tabellel.

Entscheidung zur -- Geburtshilfliche Indikation

Sektio -- Sicherheitsdenken

- Gerichtsurteile

- $\quad$ Sachverständigengutachten

- $\quad$ Einzelfallanalysen

Hausinterne Richtlinien

Qualitätssicherungskriterien

lich unter dem Durchschnitt. 2 Sanatorien liegen mit $20 \%$ erheblich darüber.

Diese Abweichungen vom Mittelwert dürften weniger durch unterschiedliche Patientenkollektive als vielmehr durch eine besonders strenge, bzw. fehlende hausinterne Kontrolle und ein in der privaten Geburtshilfe besonders ausgeprägtes Sicherheitsdenken bedingt sein.

Die Sektiorate der II. Frauenklinik halt seit 10 Jahren relativ konstant bei 12 \%.

Patientinnen und Methode

Die Daten der Entbindungen an der II. Wiener Frauenklinik sind seit 1976 vollständig mittels EDV dokumentiert (Tab. 2). 
Die führenden Sektioindikationen sind im folgenden in absolute und relative Indikationen unterteilt.

Die absoluten Indikationen wie Schädel-Becken Miß-verhältnis, Plazenta praevia, vorzeitige Lösung und dro-hende Uterusruptur machen mit knapp 20\% nur eine Minorität der Indikationen aus und unterliegen nur geringfügigen Schwankungen.

Die Mehrzahl der Sektioindikationen fällt in die Kate-gorie der relativen Indikationen und unterliegt stärkeren Veränderungen.

Die Indikation drohende intrauterine Asphyxie als häufigste Indikation zur operativen Geburtsbeendigung nimmt an Bedeutung mit den Jahren zahlenmäßig zu und soil nicht nur durch das CTG, sondern auch durch die MBU vom fetalen Skalp untermauert sein, um unnóti-ge Eingriffe zu vermeiden.

Die MBU ist eines der wirksamsten Instrumente zur Einsparung von Kaiserschnittoperationen. Postpartal soil die Richtigkeit der Indikationsstellung unbedingt durch Messung des Nabelarterien-pH über-prüft werden.

Lage- und Haltungsanomalie als zweithäufigste Indikation zeigt keine signifikante Änderung im Zeitverlauf.

Die Indikation protahierte Geburt gewinnt wesentlich an Bedeutung, anscheinend unter dem Eindruck des

hauptsächlich in der angelsächsischen Literatur propagier-ten ,active management of labor”. (O’Driscoll, 1984) [2]. Sosehr die publizierten Daten dafür sprechen, einen Ge-burtsfortschritt von $1 \mathrm{~cm}$ pro Stunde und eine Gesamt-dauer von nicht mehr als 12 Stunden anzustreben, erscheint doch im Sinne der Einsparung von Sektiones eine weniger strikte Definition des minimalen erforderlichen Geburtsfortschrittes angebracht.

Die traditionellen Indikationen wie alte Erstgebärende, dringender Kinderwunsch, Zustand nach Sterilitätsbehand-lung, beiasteie Anamnese, und Zustand nach Sektio verlie-ren mit Recht im Beobachtungszeitraum an Bedeutung.

Tabelle 2. Relative Sektioindikationen (\%)

Sektiotechnik

Nicht nur die Indikation zur Sektio, sondern auch die Sektio als Operation selbst unterliegt einem gewissen Wandel. Tabelle 3 gibt die Operationstechnik wieder. wie sie derzeit an der II. UFK geübt wird. Es soil dabei keines-wegs der Anspruch auf die optimale Technik erhoben werden. Die Laparotomie wird nur dann median ausgeführt, wenn bereits eine derartige Voroperation stattgefunden hatte, ansonsten als Pfannenstiellaparotomie, auch bei dringlicher Indikation. Die Uterotomie wird bei sehr klei-nen Frühgeburten und nicht entfaltetem unterem Uterin-segment gelegentlich isthmisch median, ansonsten immer isthmisch quer angelegt. Die zweischichtige Uterusnaht wird von der Mehrzahl der Operateure bevorzugt. Das Aufdehnen des Zervikalkanals mit Hegarstiften soil wegen seiner potentiellen Risiken nur bei fest geschlosse-nem Muttermund vorgenommen werden.

Das Abstopfen des Darmes mit Bauchtüchern ist in den meisten Fallen nicht nötig. Das Unterlassen des Abstop-fens, wie es bei Leitungsanaesthesien erforderlich ist, führt erfahrungsgemäß dazu, daß weniger Blut und Fruchtwasser in die Bauchhöhle gelangt. Diathermiegeräte sollten wegen ihrer vielfältigen Störungsmöglichkeiten bei der Sektio nicht eingesetzt werden, solange sich das Kind in utero befindet. 
Tabelle 3. Sektiotechnik

Conclusio

Die Analyse der Sektiofrequenz und der Häufigkeit einzelner Indikationen erlaubt es den einzelnen Abteilun-gen, positive, aber auch Fehlentwicklungen zu erkennen und gezielte Maßnahme zur Eindämmung der Sektiofrequenz zu setzen.

Literatur

O’Driscoll K, Foley M: Obstel Gynecol 61: 1-5 (1983).

O’Driscoll K. Foley M. MacDonald D: Obstet Gynecol 63: 485-490 (1984). 